\title{
Diagnostic Efficacy of Fine Needle Aspiration Cytology in Neck Swelling - A Systematic Review
}

\author{
Niveditha S. ${ }^{1}$, Suresh R. Chandak ${ }^{2}$, Yashwant Lamture ${ }^{3}$ \\ 1,2,3 Department of General Surgery, Jawaharlal Nehru Medical College, Datta Meghe Institute of Medical Sciences, \\ Sawangi, Meghe, Wardha, Maharashtra, India.
}

\section{ABSTRACT}

\section{BACKGROUND}

Neck swellings are one of the most common presenting complaints of patients in all age groups. Aetiology of neck swelling has various causes congenital or acquired, cystic, inflammatory or neoplastic and vascular. Our aim was to study the diagnostic efficacy of fine needle aspiration cytology (FNAC) in neck swellings.

\section{METHODS}

A systematic search on the database of PubMed, Google Scholar and Academia for studies on diagnosing neck swellings by FNAC where histopathological diagnosis is considered gold standard was done and reviewed. The keywords used for searching were FNAC, neck swellings, diagnostic efficacy of FNAC.

\section{RESULTS}

Fine needle aspiration cytological study was considered as the first line investigation for patients presenting with neck swellings and its sensitivity, specificity, positive predictive value, negative predictive value and diagnostic accuracy has been reported.

\section{CONCLUSIONS}

FNAC is easy to perform with almost nil complications and excludes the need for a biopsy which is more complex with complications like infection and haemorrhage. FNAC in neck swellings should be considered as a complementary investigation for histopathological studies, but never a substitute.

\section{KEY WORDS}

FNAC, Thyroid, Tuberculosis (TB) Lymphadenitis, Lymphoma, Secondaries in Neck
Corresponding Author: Dr. Niveditha $S$.,

Department of General Surgery, Jawaharlal Nehru Medical College, Datta Meghe Institute of Medical Sciences, Sawangi, Meghe, Wardha Maharashtra, India.

E-mail:devu.devoos@gmail.com

DOI: $10.14260 /$ jemds/2021/177

How to Cite This Article:

Niveditha S, Chandak SR, Lamture $Y$. Diagnostic efficacy of fine needle aspiration cytology in neck swelling - a systematic review. J Evolution Med Dent Sci 2021;10(11):829-834, DOI: 10.14260/jemds/2021/177

Submission 25-09-2020,

Peer Review 20-01-2021, Acceptance 28-01-2021, Published 15-03-2021.

Copyright (C) 2021 Niveditha S. et al. This is an open access article distributed under Creative Commons Attribution License [Attribution 4.0 International (CC BY 4.0)] 


\section{BACKGROUND}

Neck or cervical region, in simple words, comprises the area between the skull base and the clavicles, but anatomically, it is a complex region comprising of many vital structures including thyroid gland, parathyroid glands, lymph nodes, submandibular salivary glands, oesophagus, trachea, major neurovascular structures etc. ${ }^{1}$ Neck swellings are common presenting complaints of patients of all age groups and both genders. Most often, the swelling itself is the presenting complaint, without any additional symptoms. The differential diagnosis of neck swellings is vast. A proper knowledge about the embryology and anatomy of the neck structures is very essential in making a correct diagnosis and proper management of patients presenting with neck swellings. FNAC is an easy, safe and less expensive procedure which can be done on outpatient basis and has got almost no complications. There is no risk of seedling of tumour in cases of malignancy and there is no post procedure scar. It can even be repeated easily if required. Some of the common causes of neck swellings are as follows ${ }^{1}$

1) Congenital: thyroglossal cyst, branchial cyst, haemangioma, dermoid cyst, ectopic thyroid, thymic cyst.

2) Skin \& subcutaneous tissue: sebaceous cysts, lipoma.

3) Thyroid swellings: multinodular goiter (MNG), solitary thyroid nodule (STN), carcinoma thyroid.

4) Salivary gland swellings: submandibular sialadenitis, pleomorphic adenoma, Warthin's tumour.

5) Reactive cervical lymphadenopathy: TB lymphadenitis, reactive to other infections.

6) Malignant cervical lymphadenopathy: primarylymphoma, secondary-metastatic lymphadenopathy (squamous or adenocarcinoma).

7) Vascular neck swellings.

Fine needle aspiration studies for evaluation of neck swelling were first done and reported in 1847 by Kun. However, it was not well accepted. In 1930s, Memorial Sloan Kettering reported the use of needle biopsy for evaluation of head and neck masses, but the usage of large bore needles was associated with frequent complications, one of which was occasional tumour seeding along the biopsy tract. In 1970s, Swedish physicians signified magnificent results in cytological studies of secondaries in neck swelling using fine needle aspiration and this led to the resurgence of FNAC. Since then, FNAC of neck swelling got widely accepted and is considered as a safe, fast and cost-effective procedure for diagnosing neck swelling.

\section{Research Question}

To study the sensitivity, specificity and diagnostic accuracy of FNAC of neck swellings.

\section{METHODS}

\section{Search Strategy}

A comprehensive set of keywords or search terms were built like midline swelling, FNAC, thyroid, TB lymphadenitis, lymphoma, secondaries in neck. We searched Medline, Scopus,
Google Scholar, PubMed, Web of Science, Cochrane Central Register of Controlled Trials databases and selected relevant observational studies up to June 2020. The list of reference articles was retrieved and they were searched via automation and manually. Studies were restricted to English language and humans only.

\section{Data Extraction}

Data was extracted by 2 authors into separate forms on Microsoft Excel 2020. Article selection and data extraction discrepancies were resolved through discussion. No publication date restriction was set. Automated \& manual reduplications was performed.

\section{Inclusion Criteria}

All neck swellings in patients of all age group.

\section{Exclusion Criteria}

- Vascular swellings in neck.

- Recurrent swelling.

- $\quad$ Post radiotherapy, post chemotherapy treated swelling. Acute suppurative swelling.

\section{DISCUSSION}

Virk et al. ${ }^{1}$ study conducted in 70 patients at Department of Pathology, Adesh Institute of Medical Sciences (AIMSR), Bathinda, from 1st January 2017 to 31st December 2017 showed that FNAC diagnosis of 65 patients $(92.86 \%)$ were benign while that of remaining 5 patients $(7.14 \%)$ were malignant. On histopathology, 64 patients (91.43\%) had benign lesions and 6 patients $(8.57 \%)$ had malignant lesions. Based on this, the sensitivity, specificity, positive predictive value, negative predictive value and diagnostic accuracy of FNAC in neck swelling were $83.33 \%, 100 \%, 100 \%, 98.46 \%$ and $98.57 \%$ respectively. A high specificity rate and positive predictive value has also been observed. In this study it also concludes that FNAC should be considered as the first line investigation for neck swelling and it directs the clinician to plan the treatment for the patient.

Ahmad et al. ${ }^{2}$ study was conducted in 50 patients who presented to Postgraduate Medical Institute, Lady Reading Hospital, Peshawar with neck masses, in whom 16 were males and 34 were females belonging to the age group of 15 - 55 years. The pre-operative FNAC reports were compared with the post procedure or postoperative histopathological report. The commonest diagnosis was tuberculous lymphadenitis i.e. 18 patients (36 \%), followed by reactive or nonspecific lymphadenitis, 9 patients (18\%). Malignant neoplasms, cysts, benign neoplasms and sialadenitis accounted for 7 (14\%), 5 (10\%), 4 (8\%) and $3(6 \%)$ patients respectively. FNAC was inconclusive in 4 (8\%) of cases. Carcinomas metastasis to the lymph nodes was the most common type of malignancy followed by lymphoma and papillary thyroid carcinoma. As per the study, FNAC had a specificity of $76.5 \%$ and sensitivity of $78.1 \%$ in diagnosing cervical swelling. 


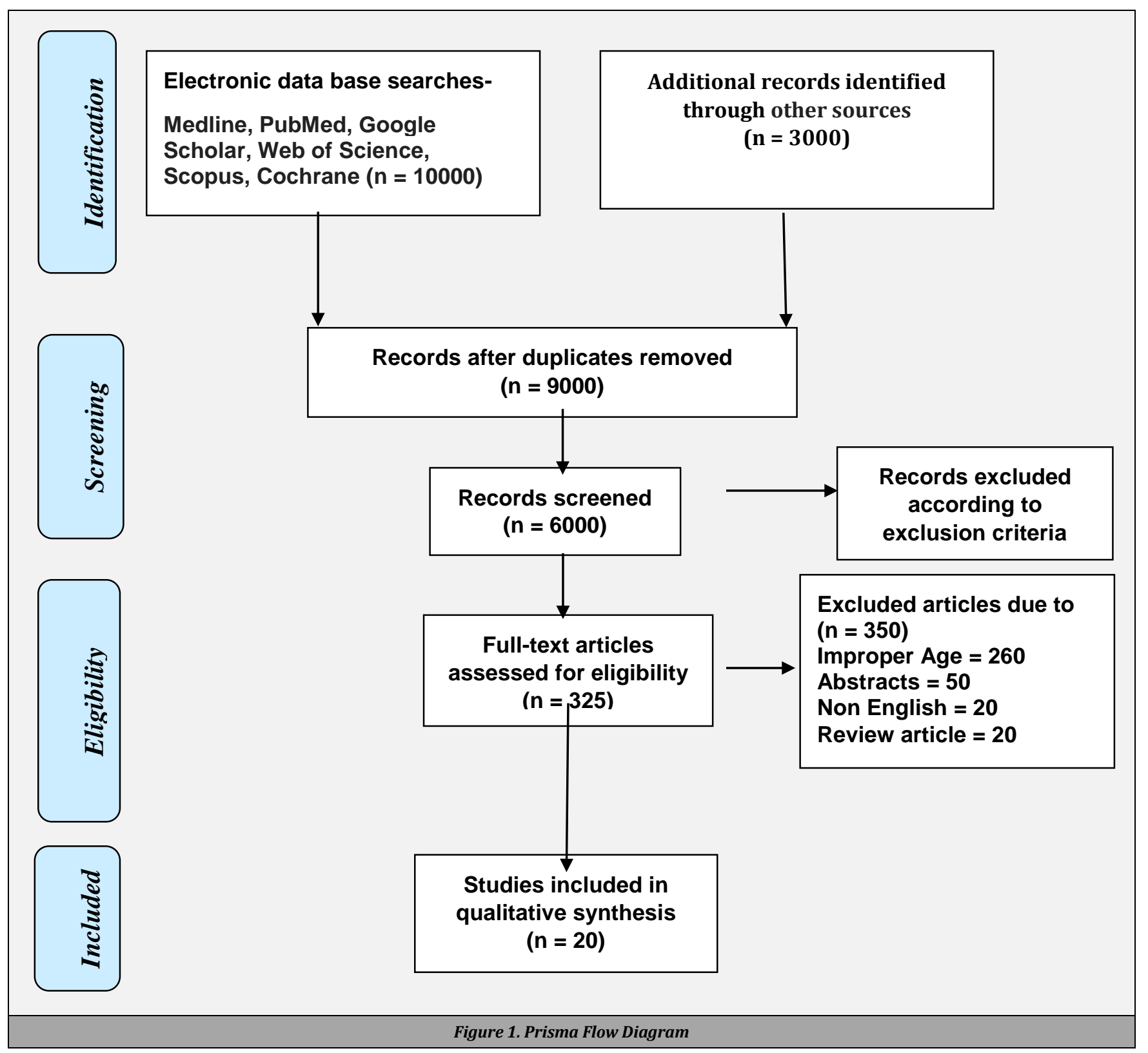

Babu K et al. ${ }^{3}$ comparative study on the diagnostic efficacy of fine needle aspiration cytology to open biopsy in cervical lymphadenopathy done in Kanchipuram district of India was conducted in 50 patients with cervical axillary lymphadenopathy revealed an overall diagnostic accuracy rate of $82 \%$ for FNAC in cases of neck swelling. The accuracy for diagnosing TB lymphadenitis was $91.70 \%$ and for secondaries was $80 \%$. Overall diagnostic accuracy for metastatic carcinoma was $80 \%$ and for squamous cell carcinoma was 100 $\%$. In the present study, accuracy was of $91.70 \%$ for tuberculous lymphadenitis. FNAC excludes the need for excisional biopsy in cases of cervical lymphadenopathy. Patients with palpable cervical lymph nodes can be easily diagnosed using FNAC. A high degree of accuracy and precision has been noted in cases like secondaries to the cervical lymph nodes mainly that of squamous cell carcinoma and tuberculous lymphadenitis. But in cases of reactive hyperplasia and non-Hodgkin's lymphoma, the distinguishing features is not well demarcated in cytological study. The highest degree of pitfall in diagnosing cervical lymphadenopathy using FNAC occurs while trying to differentiate between low grade lymphoma and reactive hyperplasia. Gold standard for diagnosis is open biopsy followed by histopathological confirmation. But the limiting factor of open biopsy is that the surgical planes will get distorted and this might increase the risk of tumour seedling especially in metastatic upper and middle cervical lymph nodes which are potentially curable with radiation therapy or lymph nodal dissection. FNAC is also very useful in cases of metastatic cervical lymphadenopathy with unknown primary as primary site can be located based on the cell type on cytology. Assessment of high-grade non-Hodgkin's lymphomas has got a high specificity rate.

Poorey et al. ${ }^{4}$ study was conducted on 179 cases, of whom majority were females (95) and males were 84. 104 cases (58.10\%) of lymphadenopathy, 35 cases (19.55\%) of thyroid swelling, 17 cases $(9.49 \%)$ of salivary gland swelling, 12 cases $(6.70 \%)$ of soft tissue swelling and 11 cases $(6.14 \%)$ of miscellaneous swellings were present in the study. The study to assess accuracy of fine needle aspiration cytology in head 
and neck masses revealed a sensitivity, specificity, positive predictive value and negative predictive value of $81.80 \%, 95$ $\%, 81.80 \%$ and $95 \%$ respectively. The efficacy or diagnostic accuracy was $92.10 \%$. Cytopathology histopathology correlation was $100 \%$ in cases of TB lymphadenitis. Out of total 179 cases, correlation between cytological and histopathological diagnosis was present in 140 cases and absent in 12 cases accounting for $78.21 \%$ and $6.70 \%$. Histopathological diagnosis for correlation with cytological diagnosis were not available in 27 cases i.e. $15.08 \%$ of total cases.

Asha et al. ${ }^{5}$ conducted a prospective study to determine the accuracy of FNAC in diagnosing neck swelling by correlating cytological diagnosis with histopathological diagnosis which is considered gold standard in 90 patients with neck swelling and it was found that majority of swellings were arising from thyroid with a female predominance, followed by lymphadenopathy and salivary gland tumours, both with a male predominance. As a whole, the sensitivity of FNAC for diagnosing malignant swelling was $64.3 \%$. The specificity, positive predictive value, negative predictive value and accuracy were $97.4 \%, 81.8 \%, 93.7 \%$ and $92 \%$ respectively.

Sahni et al. ${ }^{6}$ prospective study was about the diagnostic efficacy of FNAC in cystic lesions of head and neck region of 162 cases. Histopathology was available in only 88 cases, of which 85 cases were consistent with FNAC diagnosis. Sensitivity, specificity and overall accuracy was $85 \%, 100 \%$ and $96.59 \%$ respectively. The difficulty in performing FNAC in cystic lesions was overcome by re-aspiration from residual solid area after ultrasonography (USG) guided drainage of the cyst. In the study, histopathology was available for 35 thyroid swellings, 14 salivary gland swellings, 1 metastatic lymph node, 35 keratin cysts, 2 cold abscesses and 1 lymphatic cyst. FNAC was not correlating with histopathology in 3 cases $(2$ cases of colloid goiter with cystic change and 1 benign mucinous cyst of salivary gland). One of the major drawbacks encountered was the difficulty in differentiating metastatic squamous cell carcinoma from cystic change of an infected keratinous cyst in presence of reactive atypia, branchial cyst and Warthin's tumour with squamous metaplasia.

Muratli et al. ${ }^{7}$ study aimed at assessment of the effectiveness of FNAC in diagnosing thyroid nodules by comparison with histopathological diagnosis in 126 patients showed a sensitivity of $87.1 \%$ and specificity of $64.6 \%$. The positive predictive value (PPV), negative predictive value (NPV) and diagnostic accuracy rates were $76.1 \%, 79.5 \%$ and $77.3 \%$ respectively. The study concluded that the thyroid FNAC's with Bethesda system correlated highly with the histopathological diagnosis. It also states that combination of additional and advanced diagnostic studies like immunocytology and molecular pathology techniques enhances the prognostic value of FNAC especially in patients with atypia of undetermined significance or follicular lesion of undetermined significance, lesions suspicious for malignancy, and suspected follicular neoplasm.

In Arul et al. ${ }^{8}$ study conducted on 497 patients with cervical lymphadenopathy using FNAC, 188 cases had subsequent histopathological study. The correlation between the cytological and histological studies were done in those 188 cases. Among 188 cases, 157 (83.5 \%) were benign, 14 (7.4\%) were suspicious of malignancy, and 17 (9.1\%) were malignant as per cytological diagnosis. On correlation with subsequent histopathology, 151 cases were confirmed as benign (true negative) and 28 cases were confirmed as malignant (true positive). There were three false positive and six false negative results. The sensitivity, specificity, accuracy, positive predictive value, and negative predictive values of FNAC were $82.4 \%, 98.1 \%, 95.2 \%, 90.3 \%$, and $96.2 \%$, respectively.

Iacob et al. ${ }^{9}$ prospective cross-sectional study conducted for evaluating the efficacy and diagnostic accuracy of fineneedle aspiration cytology in evaluation of lateral cervical nonthyroid tumours, by comparison with histopathology revealed that among total 58 patients, malignancy (62\%) outnumbered benign conditions (38\%). Cytology showed a specificity of $76.5 \%$ and sensitivity of $78.1 \%$ in detecting malignant lateral neck swellings. Both the diagnostic tests showed correlation. On examination of the sample obtained by fine needle aspiration, higher efficiency was seen in differentiating inflammatory lesions from benign or malignant lesions. However, in some situations cytological data was inadequate for a proper assessment, as in cases of lymphoma, where cytology can only suggest a diagnosis. A proper and specific description can only be obtained with further histopathological and immunohistochemical examinations.

According to Sellami et al. ${ }^{10}$ retrospective study which was aimed at evaluating the cytology of cervical lymphadenopathy conducted in 131 patients revealed, the sensitivity, specificity, PPV of FNAC in diagnosing TB lymphadenitis were $83.3 \%$, $83.3 \%, 78.9 \%$ and $86.9 \%$ respectively.

Rajbhandari et al. ${ }^{11}$ study conducted in 64 patients with head and neck swellings had $61 \%$ females and $39 \%$ males with a maximum incidence of cervical lymphadenopathy (45 $\%)$ followed by thyroid swellings (37.5\%) and salivary gland swellings (16\%). An overall sensitivity of $86 \%$, specificity of $97 \%$ and accuracy of $87.4 \%$ was observed in the study, in which highest sensitivity, specificity and diagnostic accuracy was seen for thyroid swellings. The study also observed that FNAC was falsely negative for malignancy in 4 cases of nonHodgkin's lymphoma and falsely positive for malignancy in 1 case of reactive lymphadenopathy which subsequently reduced the diagnostic accuracy of FNAC in cases of reactive lymphadenopathy and non-Hodgkin's lymphoma to $77.8 \%$ and $66 \%$ respectively, however, showed $100 \%$ diagnostic accuracy in cases of TB lymphadenitis and secondaries (metastatic deposits). Overall diagnostic accuracy for FNAC in lymph nodal swelling was $82.75 \%$.

Bagga and Mahajan et al. ${ }^{12}$ study conducted in 252 patients and aimed at determining the utility and diagnostic accuracy of FNAC of thyroid swelling showed a diagnostic accuracy of $96.2 \%$, a sensitivity of $66 \%$ and specificity of $100 \%$.

In the study conducted by Devi and Talukdar et al. ${ }^{13}$ to assess the frequency of various benign and malignant neoplasms in different age group and to evaluate the diagnostic accuracy of fine needle aspiration cytology in head and neck neoplasms revealed that out of 336 cases of head and neck tumours, $48.81 \%$ of cases were lymph nodal swelling and $13.99 \%$ were thyroid swelling. Over all sensitivity, specificity, and diagnostic accuracy were $98.08 \%, 96.23 \%$, $97.46 \%$ respectively. When thyroid cases were taken into account, it was difficult to differentiate follicular adenoma and follicular carcinoma using fine needle aspiration cytology. Histopathological diagnosis was available in 20 cases. Among these cases, 19 cases were consistent and 1 case was inconsistent (false positive) with cytology. Diagnostic accuracy of FNAC for thyroid swelling alone was $95 \%$. 
Follicular neoplasm accounted for the majority of cases, commonly involving patients of 21 to 40 years. In cases of salivary gland swellings, histopathological diagnosis was available in 39 cases. Out of this 37 cases showed FNAChistopathology correlation and in 2 cases there was disparity between cytological and histopathological diagnosis. Diagnostic accuracy of FNAC for salivary gland swellings alone was $94.87 \%$. Most commonly involved organ is parotid and pleomorphic adenomas are the majority of lesions. Out of 164 cases of cervical lymphadenopathy, histopathological diagnosis was available in 75 cases. FNAC was correlating with histopathology in 74 cases and was not correlating in 1 case. In the study, metastasis of squamous cell carcinoma was found as the most commonly occurring lesion in both males and females.

Saraf et al. ${ }^{14}$ study conducted in Government Medical College, Jammu included 120 patients. The cytological findings of 96 out of 120 patients were compared with the histological diagnosis and FNAC showed an overall sensitivity, specificity and accuracy of $86.7 \%, 92.2 \%$ and $95.5 \%$ respectively. FNAC in cases of lymph nodal swelling showed $100 \%$ sensitivity, specificity and accuracy whereas it is $72.3 \%$ \& $75 \%$ sensitive, $86.6 \%$ \& $94.4 \%$ specific and $89.5 \% \& 92.5 \%$ accurate in cases of thyroid and salivary gland swellings respectively. 32 out of total 96 patients had thyroid swellings. Among the 32 patients, 4 had malignancy on final histopathological examination, but FNAC false negatively reported 2 cases as benign. 2 other cases were reported false positive (i.e. malignant) on FNAC, but later turned out to be colloid on histopathological examination. Hence, in their study, the diagnostic accuracy of FNAC for thyroid swelling was $87.5 \%$.

Suryawanshi et al. ${ }^{15}$ conducted a retrospective research study in 363 patients who presented with head and neck swellings. In 205 cases out of total 363 cases, a cytohistopathological correlation was evaluated. Lymph nodal swellings predominated thyroid swellings, followed by salivary gland swellings. An inconclusive FNAC report was obtained in $3.30 \%$ of cases. The overall accuracy rate of FNAC was $97.07 \%$ and the sensitivity, specificity, positive predictive value, negative predictive value of test was $80.00 \%$, $98.91 \%$, $88.88 \%$ and $97.86 \%$ respectively. When lymph nodal swellings are considered, the histopathology diagnosis was inconsistent with cytology in 3 cases, 1 each of reactive lymphadenitis, TB lymphadenitis and lymphoma making the overall diagnostic accuracy of FNAC in cases of lymph nodal swellings to $95.94 \% .110$ out of total 363 patients had thyroid swellings, of which histopathological diagnosis was available only for 68 cases. On the basis of this, the diagnostic accuracy or efficiency of FNAC for thyroid swelling was $97.06 \%$ and FNAC was not consistent with histopathology in 2 cases which were falsely negative for follicular adenoma and follicular carcinoma respectively.

Rout et al. ${ }^{16}$ conducted a prospective study in patients who presented with neck swelling to the ENT, Head and Neck Surgery and Pathology Departments of S.C.B Medical College, Cuttack, from April 2007 to March 2008. After initial assessment, proper history taking and clinical examination, these patients were subjected to fine needle aspiration cytology. Among these patients, those who had thyroid swellings were grouped separately and they subsequently underwent surgical management. This accounted for 76 cases. The pre-operative FNAC diagnosis of these patients were compared with post-operative histopathological diagnosis. A female predominance was seen in the study with colloid goiter as majority of cases. Correlation between fine needle aspiration cytology and histopathology was absent in 3 cases and thereby the diagnostic accuracy of FNAC in cases of thyroid swelling was $96.05 \%$. In the cases which had discrepancy, FNAC reported one colloid goiter and two follicular adenomas, which on histopathology turned out to be papillary carcinoma and follicular carcinomas respectively. Five cases of papillary carcinoma of thyroid showed FNAC and histopathology.

Rakhshan et al. ${ }^{17}$ studied a total of 178 patients who were inpatients of Loghman-Hakim Hospital, Tehran, Iran, with complaints of neck swelling. After initial evaluation of patients with neck swelling, these patients were subjected to FNAC. Subsequently excisional biopsy was done in all these cases. After obtaining final histopathological diagnosis, the patients with lymph nodal lesion on histopathology were selectively grouped. The study had $59.6 \%, 17.9 \%, 14.6 \%$ and $7.8 \%$ of reactive lymphadenitis, metastatic lymphadenopathy (secondaries), Hodgkin's lymphoma (HL), and non-Hodgkin's lymphoma (NHL) respectively. FNAC was inconclusive in 27 cases that accounts for $15.2 \%$ of the total cases. In remaining 151 cases, adequate sample was obtained on FNAC and was hence, available for comparison with histopathology. In the cases were FNAC and histopathology was compared, 133 cases showed correlation between both. Diagnostic accuracy of FNAC in this study was $88 \% .3$ benign cases were over diagnosed as malignant i.e. false positive (FP). 15 malignant conditions were under diagnosed as benign i.e., false negative (FN). Sensitivity, specificity, positive predictive value and negative predictive values were $75.8 \%, 96.6 \%, 94 \%$ and 85.1 $\%$, respectively. The study concluded that FNAC has a high accuracy or efficiency in diagnosing malignant cervical lymphadenopathy, but the incidence of false negative cases demands the need for further evaluation in the form of biopsy in FNAC diagnosed benign cases where there is a clinical suspicion of malignancy.

Khetrapal et al. ${ }^{18}$ study is a retrospective observational study conducted in 290 patients with head and neck swellings who reported to the Department of Pathology of Hamdard Institute of Medical Sciences and Research and Hakeem Abdul Hameed Centenary Hospital, New Delhi during the period from January 2011 to February 2013. The maximum number of aspirates in this study were from lymph nodal masses i.e. 185 cases $(64.1 \%$ ), followed by thyroid swelling (49 cases - 16.9 $\%)$. Skin and soft tissue lesions including scalp lesions accounted for $14.1 \%$ i.e. 41 cases and salivary gland lesions accounted for $4.1 \%$ of cases (12 cases). Minimum number of cases were oral cavity swelling i.e. 3 cases $(0.7 \%)$. Out of total 290 cases, histopathological diagnosis was available for comparison with FNAC in only $25.51 \%$ of cases (74 out of 290). Of these 74 cases, histological findings were consistent with the cytological findings in only 68 cases i.e. $91.8 \%$. Inconsistent findings were seen in only 6 cases (8.2\%). The study showed that FNAC had a sensitivity and specificity of $93.24 \%$ and $100 \%$ respectively.

\section{CONCLUSIONS}

Fine needle aspiration (FNA) is a valuable, atraumatic testing tool with good predictive precision. It has gained a pivotal role 
in the primary assessment of neck lesions. When one assumes the diagnostic disease is likely to be identified based on the clinical anatomy of a specific group of neck lesions, it is obvious that FNAC would show at least equal diagnostic complexity. When aspiration cytology is required, sufficient clinical records and other additional details must be gathered. Majority of the studies have shown that FNA study has a sensitivity more than $80 \%$, specificity and diagnostic accuracy more than $90 \%$ each. However, ultimate diagnosis should be on the basis of histopathological study. ${ }^{1,5,9}$ Histopathological diagnosis is still considered gold standard in cases of neck swelling. Correlation between cytopathology and histopathology is adequate for accurate diagnosis of neck swellings. In fact, aspiration cytology is considered complimentary or supportive to histopathology and not a substitute. ${ }^{11,12,15}$

\section{Financial or other competing interests: None.}

Disclosure forms provided by the authors are available with the full text of this article at jemds.com.

\section{REFERENCES}

[1] Virk YK, Rani E, Sharma GL, et al. Diagnostic accuracy of fine needle aspiration cytology in neck swellings. International Journal of Contemporary Medical Research 2019;6(5):E1-E3.

[2] Ahmad T, Naeem M, Ahmad S, et al. Fine needle aspiration cytology (FNAC) and neck swellings in the surgical outpatient. J Ayub Coll Abbottabad 2008;20(3):30-2.

[3] Bhaskar M, Chitra T, Babu DK. A comparative study of diagnostic efficacy of fine needle aspiration cytology to open biopsy in cervical lymphadenopathy in a tertiary care hospital, Kanchipuram District, India. Int Surg J 2018;5(12):3847-51.

[4] Poorey VK, Tyagi A. Accuracy of fine needle aspiration cytology in head and neck masses. Indian J Otolaryngol Head Neck Surg 2014;66(2):182-6.

[5] Asha CS, Roy BRS. Correlation of fine needle aspiration cytology and histopathology of neck swellings. Int J Otorhinolaryngol Head Neck Surg 2018;4(3):648-54.

[6] Sahni S, Shankar VS, Krishnappa A. Diagnostic efficacy of fine needle aspiration cytology in cystic lesions of head and neck region - a single experience at tertiary health care centre. National Journal of Laboratory Medicine 2017;6(2):P01-7.
[7] Muratli A, Erdogan N, Sevim S, et al. Diagnostic efficacy and importance of fine-needle aspiration cytology of thyroid nodules. J Cytol 2014;31(2):73-8.

[8] Arul P, Masilamani S, Akshatha C. Diagnostic efficacy of fine-needle aspiration cytology in the evaluation of cervical lymphadenopathy. J Sci Soc 2016;43(3):117-21.

[9] Iacob A, Zazgyva A, Ormenişan A, et al. Effectiveness of fine-needle aspiration cytology in the diagnosis of lateral cervical nonthyroid tumors. Medicine (Baltimore) 2016;95(31):e4448.

[10] Sellami M, Charfi S, Chaabouni MA, et al. Fine needle nonaspiration cytologyfor the diagnosis of cervical lymph node tuberculosis: a single center experience. Braz J Otorhinolaryngol 2019;85(5):617-22.

[11] Rajbhandari M, Dhakal P, Shrestha S, et al. The correlation between fine needle aspiration cytology and histopathology of head and neck lesions in kathmandu university hospital. Kathmandu Univ Med J (KUMJ) 2013;11(44):296-9.

[12] Bagga PK, Mahajan NC. Fine needle aspiration cytology of thyroid swellings: how useful and accurate is it? Indian J Cancer 2010;47(4):437-42.

[13] Devi J, Talukdar KL. Evaluation of diagnostic value of fine needle aspiration cytology (FNAC) in head and neck neoplasms with application of immunological markers in selected cases-a hospital based study to enhance early detection, diagnosis and management. IAIM 2017;4(1):20-37.

[14] Saraf A, Gupta SB, Manhas A, et al. Fine needle aspiration cytology versus histopathological examination in head and neck lesions. Journal of Dental and Medical Sciences 2016;15:122-8.

[15] Suryawanshi KH, Damle RP, Nikumbh DB, et al. Cytohistopathological correlations of head and neck swellings in a rural hospital in north maharashtra: our experience. Annals of Pathology and Laboratory Medicine 2015;2(4): A121-6.

[16] Rout K, Ray CS, Bahera SK, et al. A comparative study of FNAC and histopathology of thyroid swellings. Indian J Otolaryngol Head Neck Surg 2011;63(4):370-2.

[17] Rakhshan M, Rakhshan A. The diagnostic accuracy of fine needle aspiration cytology in neck lymphoid masses. 2009;4(4):147-50.

[18] Khetrapal S, Jetley S, Jairajpuri Z, et al. FNAC of head \& neck lesions and its utility in clinical diagnosis: a study of 290 cases. National Journal of Medical Research 2015;5(1):33-8. 